\title{
Some ideas on the teaching reform of the numerical analysis for the undergraduate
}

\author{
Shouhui Zhang \\ School of Mathematical Sciences \\ University of Jinan \\ Jinan, China \\ Email: ss_zhangsh@ujn.edu.cn
}

\author{
Xuanxin Wang \\ School of Mathematical Sciences \\ University of Jinan \\ Jinan, China \\ Email: ss_wangxx@ujn.edu.cn
}

\begin{abstract}
Numerical analysis is a mathematics course, which is applied widely in model constructing and application for engineering and techniques. Based on the teaching experience of many years, the author mainly puts forward some new thinking on the teaching reform, which includes the transform of learning concept, the adjustment of teaching methods, and the mobilization of learning enthusiasm. Combining with the development of the science computing, the paper gives some advise on bring up the ability to solving the actual problems by mathematics. It can improve the teaching validity of numerical analysis and let the undergraduate enjoy learning and hardworking on computing, which can prepare a good base for the follow-up learning and research, and can promote the reform of the textbooks and so on.
\end{abstract}

Keywords-numeircal analysis; teaching reform; numerical experiment; thinking way; applied course

\section{INTRODUCTION}

As the development of science technology, the science and engineer computing becomes more and more important for science application and is almost used in all science fields. As the computer comes into being and the high development of the information technology, there are more and more large science computing by making use of computer or parallel computer. But there is a large distance between the application and computing in computer. Numerical analysis is a necessary and important bridge between the theoretical and application by constructing some numerical methods for solving the approximation of the solution.

As a professional required course of the computing mathematics major, numerical analysis ${ }^{[1-2]}$ has its particularity, plays an important role in professional study, and takes part in exams for the graduate, so it needs more attention and learning hard. But at the beginning of learning the course, the undergraduate usually feel confused for its wide base knowledge, strong theoretical and different knowledge framework.

Based on the teaching experiments of the undergraduate and the graduate for many years, the author refine the following ideas about teaching reform and improvement by some ways, such as the course setting, character of the course and the undergraduate, in order that the students can understand and control the course much easier and establish a good foundation for the follow-up study.

\section{THE CHARACTERISTIC OF NUMERICAL ANALYSIS}

Because both the mathematics college and the computer college have a course named as numerical analysis or numerical methods, many people always misunderstand them as a same thing. But actually, they are not same for they focus on different things.

In computer college, they care what problems the software of numerical method can deal with, and how to make use it? While mathematics college focus on how to find some new numerical method which has well numerical property, and generate the respective software. So the ways of teaching and learning in different college is very different.

Although there are many kinds of numerical methods for difference problem, there is something in common. There are three important points to control, the first one is the construction of numerical method, the second one is the numerical analysis of each methods, and the last one is the numerical experiments. The first two are based on theoretical knowledge, the last is based on the computer knowledge and ability to deal with the actual problem.

In next section, according to the background of the course and the teaching experience, some ideas about the teaching reform are put forward.

\section{IDEAS OF TEACHING REFORM}

In the learning of the undergraduates who major in information and computer science, the theoretical study is the most important part for the first two years, while the most important course is numerical analysis for the next two years, for it will decide whether they have the ability to solve the actual problems by mathematic methods, which is the aim of the major setting.

In this part, the author put forward some ideas on the teaching reform ${ }^{[3-4]}$ by some different ways. 


\section{A. The aim of course}

At the first impression of this course, the students always think it's very simple, for the problems to be solved is much easier than in the advanced mathematics. But as they study it step by step, they will find that the problems has no good condition to make sure of the exact solution existing.

In another words, although the problem is simple, the old method is not suitable for solving it. But, such problems is existed widely in real life and need being solved, while the problems with exact solution in advanced mathematics is high abstracted and idealized. We named these solving procedure of the actual problems by computer as scientific computing.

As the development of science, there are more and more scientific computing comes into being. As a professional course of computing mathematics, numerical analysis needn't solve the exact solution but the numerical approximation. Some problem hasn't the exact solution for it hasn't the required precondition such as smoothness and differential, and the other's exact solution can't be solved because its exact solution is very difficult to obtain, or the exact solution exists but the expression form is too complex to be used easily.

Numerical analysis is a professional required course set in grade three of the undergraduate, and a major professional course in the exam for the graduate and the required public course of the engineering graduate. So the course not only fulfill the need of major but also is a necessary tool of making use of mathematical. Based on the learning of this course, the undergraduate can transfer to study almost engineering majors for the graduate and can apply for a jobs which will conclude numerical computing. So it is not only a course as a bridge between mathematical and science application, but also a bridge from the pure theoretical major to the applied major

\section{B. The preparation of numerical analysis}

The major research focus is the numerical computing in the advanced mathematics, so it needs many relative precourses, and makes use of lots of formula which is difficult o remember.

For the course aims at the solving of the problems in the advanced mathematics by computer, the learning will needs not only the theoretical knowledge in the advanced mathematics, advanced algebra, ordinary difference equation and so on, but also the knowledge of computer language ${ }^{[7-8]}$, such as $\mathrm{C}+$, Fortune, Matlab and so on.

So the course need that the students prepare all the knowledge well. The corresponding teaching should conclude some review about the respective knowledge, which will make the students accept the new knowledge much easier.

As the solution is an approximation to the exact solution, beside of the numerical computing, it needs a whole numerical analysis and the corresponding algorithm research. So in this course we need to improve the numerical computing method step by step and get some more and more precise numerical solution.

Then the numerical analysis is a course full of improvement and up-to-date. So the students needs to be guided to the way of thinking that is full of the rapidly changing numerical methods and more and more large scientific computing comes into being.

So before the beginning of learning the new knowledge, the teacher should review the respective basic knowledge, guide the thinking way of this course, show the aim and the meaning of the course to the students, that will stable the confidence and mobilize the interesting of learning.

Among the introduction of the numerical method and numerical analysis, the undergraduates should be guided to find the ideas of the construction and the theoretical way to analyze the numerical property. By then, they can bring up the consciousness of dealing with the actual problem by numerical computing .

The numerical experiments should be paid more attention because it can not only mobilize the learning interesting of the undergraduate, but also can discover much more ideas on how to improve the property of the numerical methods. By writing amount of programs and debug them, the undergraduate can understand the construction of the numerical methods well and make use of them more flexibly.

\section{The establishment of consciousness for numerical analysis}

Numerical analysis is a course which is not only a very important course for the reality but also is a no ending course, because the numerical solution can be improve to be more and more precise to the exact solution but never reach it. But for the students who are accustomed to find the exact solution, it's difficult to mobilize the interesting to find the numerical solution and analysis the theory property.

So the first difficulty for the student is the transform of the thinking way from solving the exact solution to the numerical solution. Although they end the theory study just now, the undergraduate still meet some difficulty in learning this course Because in the numerical analysis, the solution is uncertain.

At the same time, the solving and theory analysis of numerical solution is a more difficult job which needs combining more knowledge and has a different thinking way from solving the theory solution. In addition, there is a new part named as error lying along with the numerical solution, so the theory analysis is necessary for numerical methods.

As the course is very important and difficult to be controlled for the undergraduate, it is necessary for the teacher to reform the teaching way in order to let it easy to be accepted, understood and controlled by the students. Based on the teaching experience of many years, the author put forward the following ideas about the reform.

\section{The reform of teaching}

In this part, some ideas about the teaching reform are proposed.

- Mobilizing the students' interesting by the wide application of numerical analysis

The teacher should put forward of many application examples in reality before the teaching of numerical 
algorithm, by then the students can understand the reason of solving not the exact solution but the approximation, then realize the meaning to find the numerical solution to instead of the exact solution.

From these actual problems, the students can realize that the known condition of problems in advanced mathematics always don't exist in the reality, such as smoothness and difference enough; then they know even if they has learned the theoretical knowledge for two years, they still can't solve the actual problem. So finding some new methods to solve these problem is very important for application and engineering production.

On the other way, by seeing the numerical solution solved by the numerical algorithm visually for the familiar reality problem, the interesting of learning is mobilized easily and students would like to solve such similar problem in reality which will improve their self-confidence to study this course.

- Paying more attention on the idea of construction by simplifying the teaching content

As it is well known, numerical analysis is a bridge between mathematics and the application, so the major aim of numerical analysis is the application of mathematics in reality. Then we could pay more attention in understanding the construction of some basic numerical method and making use of them, not need to care the details of each method, for it's too much and complex to master.

At the same time, it's necessary for the undergraduate to master the idea of construction and improvement way of each basic numerical methods, because it's the main difference between the computing mathematics and the computer science, for the computer science only focus on the application of the numerical method, not care the theoretical analysis, so they only make use of the existing numerical methods. This is the main reason that the information technology would like to offer the job to the undergraduate of computing mathematics.

So the students can study this course hard and master the tools well, in order to improve one's competitive power and prepare the exam to the graduate well.

- Improving the ability to deal with problem by making use of numerical computing

After understanding the importance of the course and the main content, the next task is to face the course and learn it. Instead of the theoretical knowledge, the study focus of this course conclude not only the theory but also the application.

So when a new numerical method will be taught, some steps should be dealt with one by one. First, the respective application problem should be given, which can mobilize the interesting of the students; secondly, the teacher should lead the students to find the known condition hidden in the background and extract the mathematical model, which is the problem we will solve. This two steps are the background of the application.

Next, we begin studying on the mathematical model. According to the condition we have found and the background, we should derive what is the solution and how to obtain it, which is the constructing procedure of the numerical method and will be taught in detail. Usually, there is not the exact solution, so when we find the way to solve it, it's necessary to face the approximating and cancelling some little influence factors, which will cause the final error lied in the numerical solution to the exact one.

When we obtain a numerical solution by the method, we want to know the difference between the numerical solution and the exact solution, but the exact solution is always unknown, so we put forward some prior error estimate methods and some numerical property to make sure the effective of the numerical method. This part is the other important section of learning.

After we do all the upward steps, the last one is to prove the validity by some examples, which is the most interesting part of the learning. This is the other focus of learning the course for the students, and it needs more numerical calculating in computer and careful analyze the theoretical property and proving them by some reality examples.

Based on the basic numerical methods ${ }^{[5-6]}$, we will improve the numerical method from some ways, such as the accuracy, computation account, data storage and the complexity of the algorithm and so on.

Until now, a total learning procedure of a new numerical method is over. Besides of the above all, the teaching should get well along with the new development of science computing, which form the main part of every numerical method's implication.

- Improving the examination method

The aim of learning the course is not only getting a good score , but also improving the ability of solving problems by learning the course. So the ability of making use of the numerical method will become the main part of the examination step by step.

So the way of exam should conclude two means: one is the theoretical knowledge, the other is the ability of solving the problems by making use of the numerical method. This needs the teacher paying more attention to the students learning in usual time, from the handwork to the writing and debug of the computer algorithm. By then, the way of exam will be effect to the student.

- Improving the teaching material 
As the gradual implementation of the above reform, the teaching and the learning will meet some new problems to deal with, When these problems are solved, we can get some new experience on how to teach better and learn better. So the teaching material can be revised and compiled based on the accumulated experiments, such as more new application examples, more simplify introduction of numerical method and basic numerical analysis in detail.

And as the development of the science computing and the society production, there will be more and more new scientific computing and numerical methods comes into being, which will give much new challenge to the numerical methods, which will form the other part of the course. We can combine the classic methods with the new one to find the new ideas to update the new one.

This is a course which fill with the development and application as the society develops ${ }^{[9]}$. So it's a good course to the undergraduates to produce a thinking way filling with development and applying their knowledge in actually, but not care the present only. There will be more and more problems happened in the teaching and learning, and the reform teaching and the teaching material should be better and better as we deal with such problems one by one.

As a course set in the mathematics school, the training objectives are the thinking way of logical and the ability to solve problem. The undergraduates can develop a mathematics thinking way and have a conscious to solve the actual problems by the mathematics tools.

\section{CONCLUSION}

As an application course, the teaching of numerical analysis must focus on both the theory knowledge and the development of application at the same time. The development of the new numerical methods and computer knowledge will cause some new ideas to construct much more new numerical methods comes into being.

So the reaching reform need considering the balance between the knowledge base of the undergraduates and the teaching way, between the theoretical and the application, between the classical and new development, in order to be understood easily.

Its teaching reform is very important for all students' learning and the subject's development, which will cause more advantage in applying to the science computing and industrial production. And the teaching and learning always be the two means of the course and will improve and promote each other at the same time.

By implementing the ideas of teaching reform to the actual teaching, the students will understand and master the course much easier and bring up the consciousness of solving the actual problems by mathematics, which is the important meaning of learning mathematics.

\section{ACKNOWLEDGMENT}

S.H. Zhang was supported and was supported by Promotive Research Fund for Excellent Young and Middleaged Scientists of Shandong Province (BS2013NJ016) and the Project-sponsored by SRF for ROCS, SEM.

\section{REFERENCES}

[1] Boying Wu ed., The principle of numerical analysis[M], Science press 2003.

[2] Qingyang Li, Nengchao Wang, Dayi Yi, Numerical analysis (Fifth Edition). Tsinghua University press, 2008.

[3] Weiguo Li, Shengtian Zhou, Xinhai Liu. New exploration of teaching reform on the public degree course of the postgraduate[J], Chinese university education, 2013(3),75-77.

[4] Dacheng Wang. The research of teaching reform and the practice on the numerical analysis course for the Master postgraduate[J]. Postgraduate Education, 271-272.

[5] Renhong Wang. Numerical approximation principle[M]. Shanghai Science Press, Shanghai, 1980

[6] Kang Feng, etc. Numerical mathods[M]. National Defense Industry Press,Beijing, 1978.

[7] John H. Mathews, Numericla methods using Matlab[M]. Electronic Industry Press, 2010.

[8] Youqian Huang, Shijie Cheng, Zheoeng Chen, Numerical Experiments[M]. Beijing: Higher Education Press, 1989.

[9] A. Quarteroni, R. Sacco, F. Saleri,Numerical Mathematics[M]. New Yor k,Springer-Verlag, 2000. 